Accepted manuscript of J. Chromatogr A. 1483 (2017) 80-85

http://dx.doi.org/10.1016/j.chroma.2016.12.063

\title{
Effect of axial temperature gradient on chromatographic efficiency under adiabatic conditions
}

\author{
Krisztián Horváth*, Szabolcs Horváth, Diána Lukács \\ Department of Analytical Chemistry, University of Pannonia, Egyetem utca 10, H-8200 Veszprém, Hungary
}

\section{Abstract}

The effect of axial temperature gradient on the chromatographic efficiency was studied under adiabatic conditions by a modeling approach. The Equilibrium-Dispersive model of chromatography was used for the calculations. The model was extended by taking into account the axial temperature gradient. The results show that due to the temperature gradient, there are both retention and migration velocity gradients in the column. Since the retention factor, $\underset{\sim}{k}$ is not constant in the column, these $k$ cannot be calculated as the ratio of net retention and hold-up times. As a result of the gradual increase of migration velocity, the retention times of solutes decreases as the slope of temperature gradient increases. In addition, the band in the column have extra broadening due to larger migration velocity of the front of band. The width of bands becomes larger at larger change of temperature. In the same time, however, the release velocity of the compounds from the column is increasing as $\Delta T$ increases. Accordingly, an apparent peak compression effect makes the peaks thinner. As a result of the two counteracting effects (peak expansion, apparent peak compression) the column efficiency does not change significantly in case of axial temperature gradient under adiabatic conditions. The resolutions, however, decreases slightly due to the decrease of retention times.

Keywords: temperature gradient, separation efficiency, adiabatic conditions,

7 Equilibrium-Dispersive model

\footnotetext{
*Corresponding author, email: raksi@almos.uni-pannon.hu
} 


\section{Introduction}

One of the few possibilities of improving the efficiency of chromatographic separations is the use of fine particles [1]. By using them, fast mass transfer and high throughputs can be achieved in analytical laboratories. During the last decades, an active development of packing materials was undertaken. As a result, the size of a typical particle used in ultra-high performance liquid chromatography (UHPLC) decreased from 5.0 to $1.7 \mu \mathrm{m}$. Recently [2], a series of very small core-shell particles (1.0-1.4 $\mu \mathrm{m})$ were tested including the smallest commercially available $1.3 \mu \mathrm{m}$ core-shell phase 22$\}$. Compared to a $1.7 \mu \mathrm{m}$ fully porous phase, $20-40 \%$ gain in efficiency were observed by using the $1.3 \mu \mathrm{m}$ particles. For an UHPLC stationary phase, the price of the improved separation power is the necessity of high pressure. The viscous friction of the mobile phase pushed through the chromatographic bed causes enormous resistance to the flow and requires high inlet pressures [3]. Depending on the length of column, and the viscosity of mobile phase, operating columns packed with sub- $2 \mu \mathrm{m}$ particles often requires pressures up to 1000-1200 bar. Due to the law of conservation of energy, the energy applied to motion finally converts to heat. As a result, both radial and axial temperature gradient form in the column that affect the overall column performance.

In the middle of 70s, Halász et. al studied [4] the limits of high performance liquid chromatography. They concluded that temperature gradients existed in axial and radial directions inside the column due to the frictional heat. As a consequence, they limited the pressure drop, $\Delta p, 500$ bar in practical measurements. It was also predicted that the minimum particle size in HPLC would be between 1 and $3 \mu \mathrm{m}$. In the last four decades, several authors studied [5-11] the formation of axial and radial temperature gradients both experimentally and theoretically.

The main conclusion is that the radial distribution of the temperature causes a radial viscosity gradient of the mobile phase. The eluent is more viscous in the colder region at the column wall than in the warmer central region. As a result, the velocity of mobile phase also has a radial gradient. It flows faster in the central region of the column than close to the colder wall. Consequently, the shape of bands become parabolic, which decreases of the apparent column efficiency. Since retention depends on temperature, the radial temperature gradient causes also a radial distribution of retention factors. The adsorption equilibrium constants decrease with increasing temperature. Retention of solutes is smaller in the column center than near its wall. As a result, the migration velocity of solutes relative to the eluent velocity is higher at the col- 
umn center than at the column wall. This phenomenon further decreases column performance. Several theoretical approaches were used for simulating the effect of radial temperature gradient on column efficiency $[3,8,12-16]$. These works confirmed the deteriorating effect of the radial temperature gradient through both the flow pattern and retention change.

A possible solution to overcome the negative effect of radial temperature gradient is the decrease of efficiency of column thermostat or perfect insulation of the column. It was shown [17] that the decrease in chromatographic performance is larger in case of water bath than using a still air heater. The effect of viscous heat generation can be minimized when the temperature of the column wall is not controlled and the wall remains in contact with still air [18]. The radial temperature gradient can be diminished or eliminated by insulating the column. Recently, Gritti et. al $[19,20]$ developed a cylindrical vacuum chamber in order to isolate thermally the chromatographic column from the external air environment and to maximize resolution power in ultra-high performance liquid chromatography. It was shown that less than $1 \%$ of the viscous heat was dissipated to the external air environment. As a result, the amplitude of the radial temperature gradient is reduced $0.01 \mathrm{~K}$. Improvement in resolution power was observed due to the uniform distribution of the flow velocity across the column diameter. The eddy dispersion term in the van Deemter equation was reduced by $0.8 \pm 0.1$ reduced plate height unit, that is a significant gain in column performance.

For the estimation of separation efficiency of a non-uniform column (varying diameter, adsorption strength, flow rate, etc.) a general equation was derived by Giddings [21]. It was shown that the apparent height equivalent to a theoretical plate, $\bar{H}$, was affected by both the variations of the local $H$ s and migration velocities. For the calculation of $\bar{H}$, a simple equation was derived. The effect of axial temperature and pressure gradients on column efficiency was studied by Neue and Kele [22]. The authors analyzed the coefficients of the van Deemter equation under the idealized condition of complete radial uniformity. It was found that for an adiabatic column, the overall separation efficiency was not affected significantly up 1000 and even 2000 bar pressure drops. The authors neglected the effect of retention variation in the column completely.

The aim of this work is the study of effect of axial temperature gradient on the migration and spreading of solute zones and on the efficiency of chromatographic separations through axial the change of retentionin adiabatic cases. In this study, theoretical models provide more 
accurate insight to the chromatographic processes take place in the column than the practical 71 measurements, since in the latter case other effects can modify the column efficiency as well. 72 The Equilibrium-Dispersive, ED, model [1] was used for the simulation of chromatographic 73 runs. The effect of temperature on column efficiency was neglected. By applying the ED 74 model, efficiencies of separations for different linear temperature gradients are analyzed. 


\section{Theory}

\subsection{Effect of temperature on retention}

The dependence of the retention factor, $k$, of a compound on the temperature can be described by the following equation.

$$
k=k_{\infty} \exp \left(-\frac{\Delta H}{R T}\right)
$$

or in logarithmic form

$$
\ln k=-\frac{\Delta H}{R T}+\ln k_{\infty}
$$

where $\Delta H$ is the change of molar enthalpy of the system during adsrorption, $R$ the universal gas constant, $T$ the absolute temperature, and $k_{\infty}$ the retention factor of the solute at infinite temperature. $k_{\infty}$ is constant and it consists of the change of molar entropy and the phase ratio of the column. Eq. (2) allows the calculation of the local retention factor in the knowledge of temperature gradient, $T(z)$.

The retention time of the solute can be calculated by the following integral

$$
t_{\mathrm{R}}=\int_{0}^{L} \frac{u_{0}}{1+k(z)} \frac{1+k(z)}{u_{0}} \mathrm{~d} z
$$

where $u_{0}$ is the linear velocity of the eluent, and $L$ the column length.

\subsection{Peak formation}

In HPLC, separations take place in space, while the chromatogram is obtained in time dimension. A chromatographic peak is generated in the following steps:

1. generation of initial zone,

2. separation,

3. generation of chromatogram.

The initial solute zone is generated after the injection of a sample plug at the head of chromatograhic column. The width of the initial solute zone can be calculated as

$$
\Delta z=t_{\text {inj }} \frac{u_{0}}{1+k_{\text {in }}}
$$


where $\Delta z$ is the width of the zone of solute after injection, $u_{0}$ the linear velocity of the eluent, $k_{\text {in }}$ the retention factor of solute at the column inlet, and $t_{\text {inj }}$ the injection time.

$$
t_{\text {inj }}=\frac{V_{\text {inj }}}{F}
$$

where $V_{\text {inj }}$ is the injection volume, and $F$ the flow rate of eluent.

In Eq. (4), the conversion factor between time and space is the velocity of the zone. Accordingly, the initial width of the zone is smaller if $k_{\text {in }}$ is larger, and vice versa.

During the migration through the column, the solute band changes its shape due to mass transfer kinetics. If the retention factor of the solute has a gradient in the column, additional peak expansion or peak compression can affect the zone width depending on the relative velocities of the front and back of the zone.

$$
\begin{aligned}
& u_{\text {front }}=\frac{u_{0}}{1+k_{\text {front }}} \\
& u_{\text {back }}=\frac{u_{0}}{1+k_{\text {back }}}
\end{aligned}
$$

If $u_{\text {front }}>u_{\text {back }}$, or in other words $k_{\text {front }}<k_{\text {back }}$, extra band broadening takes place in the column. The zone widens more than it should be due to the classical band broadening effects. When $k_{\text {front }}$ is larger than $k_{\text {back }}$, however, the zones become thiner due to peak compression.

Finally, the zones leaves the column with the release velocity, $u_{\text {rel }}$, and appears on the chromatogram in time scale. The conversion between the space and time dimensions is $u_{\text {rel }}$.

$$
\Delta t_{\text {peak }}=\Delta z_{\text {final }} \frac{u_{0}}{1+k_{\text {rel }}} \frac{1+k_{\text {rel }}}{u_{0}}
$$

Accordingly, for a given final zone width, $\Delta z_{\text {final }}$, the peak on a chromatogram is thinner if the release velocity is large, and the peak is wider if $u_{\text {rel }}$ is small.

Eqs. (4) - (7) suggest that the width of a chromatographic peak is affected significantly if there is a gradient of retention factor in the column. The equations, however, did not tell anything on the overall column efficiencies.

\subsection{Equilibrium-Dispersive model}

Under linear conditions, when concentration of analyte injected onto the column is small and the rate of adsorption/desorption is infinitely high, the differential mass balance of the 
solute $[1,21]$ can be written as:

$$
\frac{\partial c(z, t)}{\partial t}+\frac{\partial k(z, t) c(z, t)}{\partial t}=-u_{0} \frac{\partial c(z, t)}{\partial z}+D_{\mathrm{a}} \frac{\partial^{2} c(z, t)}{\partial z^{2}}
$$

where $u_{A}$ is the migration velocity of the zone

$$
u_{A}=\frac{u_{0}}{1+k(z)}
$$

By Eq. (11), the effect of temperature gradient on the chromatographic separation can be simulated and studied rather accurately. 


\section{Experimental}

For the numerical solution of Eq. (11), the Martin-Synge algorithm [23] was used. The algorithm mimics the Martin-Synge plate model, i.e. a chromatographic system that is discrete in space and continuous in time. The column is divided for $N$ number of continuous flow mixers, where $N$ is the number of theoretical plates. In each plate, the following ordinary differential equation was solved with high accuracy.

$$
\frac{\mathrm{d} c_{i}[t]}{\mathrm{d} t}=-u_{A} \frac{c_{i}[t]-c_{i-1}[t]}{\Delta z}
$$

where $i$ represents the rank of the plate, $(0 \leq i \leq N) . c_{0}$ and $c_{N}$ are the injection and elution profiles, respectively. The band dispersion is taken into account by the proper choice of $N$. The algorithm can be extended for non-linear conditions, and was used successfully in the solution of different chromatographic projects [24-27].

The calculations were performed using a software written in house in Python programming language (v. 3.5, Anaconda Python Distribution), using the NumPy, SciPy and Numba packages. The following parameters were set during the calculations:

- column length, $L, 5 \mathrm{~cm}$,

- column plate number, $N, 1000$,

- eluent linear velocity, $u_{0}, 5 \mathrm{~cm} / \mathrm{min}$,

- retention factor at infinite temperature, $k_{\infty}, 0.165$,

- column head temperature, $T_{\text {in }}, 193-293 \mathrm{~K}$,

- change of molar enthalpy, $-\Delta H, 5000-15000 \mathrm{~J} / \mathrm{mole}$ (101 levels, stepsize: $100 \mathrm{~J} / \mathrm{mole}$ ), - total rise of temperature, $\Delta T, 0-50 \mathrm{~K}$ (101 levels, stepsize: $0.5 \mathrm{~K})$.

It was shown [3] that the axial temperature gradient is close to linear in case of adiabatic conditions. Accordingly, linear axial temperature gradient was applied during calculations.

$$
T(z)=T_{\text {in }}+\Delta T \frac{z}{L}
$$


Note that 1000 plate numbers was set during the calculations. The dependency of results on the number of theoretical plates was tested at different $N$ s up to 10000. 30000. The same numerical results were observed at each case.

$50 \mathrm{~K}$ increase of temperature can be generated by $\sim 1500$ bar pressure drop in case of methanol, or by $\sim 2500$ bar pressure drop in case of water. The latter is far beyond the capacity of the state of the art UHPLC systems. With certain UHPLC systems, even 1300 bar pressure can be generated that can heat methanol up by $45 \mathrm{~K}$. Accordingly, $\Delta \mathrm{T}$ was maximized at $50 \mathrm{~K}$.

The source code of the Python program can be downloaded from the supplementary materials. 


\section{Results and discussion}

\subsection{Retention behavior under axial temperature gradient}

According to Eq. (2), the retention factor of a solute changes gradually in the column if there is axial temperature gradient. In Fig. 1, the values of retention factor of a solute can be seen at different positions in the column at different temperature gradients. The change of molar enthalpy, $\Delta H$, of the compound was set to $-10 \mathrm{~kJ} / \mathrm{mol}$. It can be seen that the retention factor decreases in the column significantly due to the temperature gradient. At $10^{\circ} \mathrm{C}-\mathrm{K}$ total temperature rise, the decrease of retention factor is just slightly more than $10 \%$. At $50^{\circ} \mathrm{C} \mathrm{K}$, however, it becomes almost 50\%. For a compound with higher or lower $\Delta H$ the change of retention factor is more or less significant, respectively. An important consequence of this phenomenon is that retention factors of solutes cannot be calculated as the ratio of net retention time and hold-up time when the axial temperature gradient is not negligible. It holds for pressure drops larger than 600 bar typically.

Eq. (12) shows that the local migration velocity depends on the local retention factor. It can be seen in Fig. 2 that the local migration velocities increase gradually in the column in case of an axial temperature gradient. The level of increase depends on the total axial change of temperature. The relative migration velocity increases by $10-70 \%$ as $\Delta T$ increases by $10-$ $50^{\circ} \mathrm{C}$, respectively. It is important to note that the release velocity is always higher than the initial velocity in case of a positive axial temperature gradient. The migration of compounds shows acceleration during analysis.

As a result of the migration velocity gradientEg. (3) suggests, the retention times of solutes affected by the axial temperature gradient as well as Eq. (3) suggestsdue to the gradually varying migration velocities. In Fig. 3, the retention times can be seen as a function of $\Delta H$ and $\Delta T$. The figure highlights that the relative decrease of retention times are higher at larger $\Delta T$ values. The increasing $\Delta H$ makes the compound more sensitive toward the axial temperature gradient.

\subsection{Peak formation under axial temperature gradient}

As it was shown in the Theory section, peak formation is affected not just by the mass transfer kinetics but the gradient of migration velocity as well. First, depending on the sign of the derivative of velocity gradient, zone compression or zone expansion can occur in the column 
during migration. In case of a rising axial temperature, the slope of the velocity gradient is positive. As a result, the fronts of the peaks always move faster than the back parts. Accordingly, the zones at the end of the column become wider than they would normally be due to the mass transfer kinetics. In Fig. 4, the physical width of solute zones at the end of the column can be seen. Fig. 4 concludes the previous reasoning. The zones become wider if $\Delta T$ is larger. This effect is more significant at larger $\Delta H$ values than at smaller ones.

The zone expansion in the column does not necessarily results in wider chromatographic peaks. As it was shown by Eq. (8), peak widths affected also by the release velocity, $u_{\text {rel }}$. In case of a positive temperature gradient, the release velocities are always larger than any migration velocity in the column. Fig. 5 shows the release velocities of solutes from the column at different $\Delta T$ and $\Delta H$ values. Significant increase in $u_{\text {rel }}$ can be observed at larger temperature changes than at smaller ones. As in the case of zone widths, the change in release velocity increases as $\Delta H$ increases (becomes more negative).

It is important to note, that the release velocities are affected more significantly than the zone widths, suggesting that the chromatographic peaks become thinner due to the positive axial temperature gradient. Fig. 6 confirms this phenomenon. It can be seen that the chromatographic peaks can be thinner as the axial temperature change increases. This effect is more significant at larger (more negative) $\Delta H$ values.

\subsection{Effect of axial temperature gradient on chromatographic efficiency}

In the previous section it was shown that the chromatographic peak widths decreases due to the axial temperature gradient in the column (see Fig. 6). Fig. 3, however, showed that the retention times decreases similarly to the peak widths. Since the number of theoretical plates depends on the ratio of these two measures, it can be predicted that the apparent number of theoretical plates are not affected by the axial temperature gradient. Fig. 7 confirms this prediction. It can be seen that the number of theoretical plates do not change significantly due to the axial temperature gradient. Even at the most extreme case $\left(\Delta H=-15 \mathrm{~kJ} / \mathrm{mol}_{2} \Delta T=50 \mathrm{~K}\right)$, the loss of apparent efficiency is less than 6\%. Accordingly, by insulating a chromatographic column, one can keep its efficiency.

Giddings [21] derived a simplified equation for the calculation of apparent efficiencies in case of non-uniform column. When the efficiency is constant throughout the column, the relative efficiency can be calculated as: 


$$
\frac{N_{\text {app }}}{N}=\frac{\left(\frac{1}{L} \int_{0}^{L} \frac{1+k}{u_{0}} \mathrm{~d} z\right)^{2}}{\frac{1}{L} \int_{0}^{L}\left(\frac{1+k}{u_{0}}\right)^{2} \mathrm{~d} z}
$$

where $N_{\text {apr }}$ is the apparent number of theoretical plates. In Eq. (15), $k$ can be calculated by Eq. (1).

The differences between the results presented in Fig. 7 and calculated by Eq. (15) were less than $0.2 \%$ in each case (less than $0.049 \%$ in average). It confirms the validity of results presented in Figs. 1-7 and the proper choice of calculation parameters.

In chromatography, analyst need resolution not plate number. It is well known that the chromatographic resolution, $R_{s}$, depends on the number of theoretical plates, $N$, the difference and the sum of retention times.

$$
R_{s}=\frac{\sqrt{N}}{2} \frac{\Delta t_{\mathrm{R}}}{t_{\mathrm{R}, 1}+t_{\mathrm{R}, 2}}=\frac{\sqrt{N}}{2} \frac{\alpha-1}{\alpha+1+\frac{2}{k_{1}}}
$$

where $k_{1}$ is the apparent retention factor, and it is defined as $\frac{t_{\mathrm{R}, 1}-t_{0}}{t_{0}}$, and $\alpha$ is the apparent selectivity, that is the ratio of the apparent retention factors.

Close examination of Eq. (16) and Fig. 7 highlights that the resolutions in case of axial temperature gradients are not affected through the change of number of theoretical plates. $R_{s}$, however, can be affected through selectivity, $\alpha$, and retention, $k_{1}$. Depending on the difference of molar changes of enthalpy, $\Delta H \mathrm{~s}$, of the two compounds, the selectivities can also be improved and deteriorated. If $\Delta H$ of the first eluting compound is larger than that of the second one, its retention time decreases more than that of the first one (see Fig. 3). As a result, $\alpha$ increases due to the axial temperature gradient. If $\Delta H$ of the second compound is larger, $\alpha$ decreases. Accordingly, a general rule cannot be stated for how selectivities change due to the axial temperature gradient. Fig. 3, however, showed that retention times can change significantly due to the axial temperature gradient. Therefore, resolutions can be decreased through the retention. It is important to note however, that the decrease of $R_{s}$ depends on the absolute value of $k_{1}$ not its relative decrease. Eq. (16) is a concave saturation function (just like Langmuir isotherm). Its derivative is 


$$
\frac{\mathrm{d} R_{s}}{\mathrm{~d} k_{1}}=\frac{\sqrt{N}(\alpha-1)}{\left[2+k_{1}(\alpha+1)\right]^{2}}
$$

that is always positive and continuously decreasing. As a consequence, the decrease of retention has more significant effect if $k$ is small. This phenomenon can be seen in Fig. 8. The figure shows clearly that a modest decrease in resolution can be observed due to the axial temperature gradient. The degree of resolution loss is negligible. Even in the most extreme case it is less than $10 \%$, that is less than the accuracy of determination of $R_{s}$ according to our experience. 


\section{Conclusions}

The formation of a chromatographic peak, including its final position and shape, is affected by the retention of the solute significantly. In case of a linear axial temperature gradient, the front of the solute zones migrate faster than their rear part. As a result, extra peak broadening takes place in the column. In the same time, however, the high release velocities compensates this broadening practically. Neither the number of theoretical plates nor the resolutions are affected significantly by the axial temperature gradient. It means, that by insulating a chromatographic column, one can keep its separation power. Because of the gradual change of retention factors, the measure ealculates calculated as the ratio of net retention $\left(t_{R}-t_{0}\right)$ and hold-up times $\left(t_{0}\right)$ is not the retention factor and does not have any significant physical meaning.

\section{Acknowledgment}

This work was supported by the Hungarian Scientific Research Fund (OTKA PD 104819). Krisztián Horváth acknowledges the financial support of the János Bolyai Research Scholarship of the Hungarian Academy of Sciences. 


\section{References}

[1] G. Guiochon, A. Felinger, D. G. Shirazi, A. M. Katti, Fundamentals of Preparative and Nonlinear Chromatography, Academic Press, Amsterdam, 2006.

[2] A. Sanchez, G. Friedlander, S. Fekete, J. Anspach, D. Guillarme, M. Chitty, T. Farkas, Pushing the performance limits of reversed-phase ultra high performance liquid chromatography with $1.3 \mu \mathrm{m}$ core-shell particles, J. Chromatogr. A 1311 (2013) 90-97.

[3] K. Kaczmarski, F. Gritti, G. Guiochon, Prediction of the influence of the heat generated by viscous friction on the efficiency of chromatography columns, J. Chromatogr. A 1177 (2008) 92-104.

[4] I. Halász, R. Endele, J. Asshauer, Ultimate limits in high-pressure liquid chromatography, J. Chromatogr. A 112 (1975) 37-60.

[5] H. Poppe, J. Kraak, J. Huber, J. van den Berg, Temperature gradients in HPLC columns due to viscous heat dissipation, Chromatographia 14 (1982) 515-523.

[6] T. Welsch, M. Schmid, J. Kutter, A. Kálmán, Temperature of the eluent: A neglected tool in high-performance liquid chromatography?, J. Chromatogr. A 728 (1996) 299-306.

[7] A. Brandt, G. Mann, W. Arlt, Temperature gradients in preparative high-performance liquid chromatography columns, J. Chromatogr. A 769 (1997) 109-117.

[8] A. de Villiers, H. Lauer, R. Szucs, S. Goodall, P. Sandra, Influence of frictional heating on temperature gradients in ultra-high-pressure liquid chromatography on $2.1 \mathrm{~mm}$ i.d. columns, J. Chromatogr. A 1113 (2006) 84-91.

[9] F. Gritti, G. Guiochon, Complete temperature profiles in ultra-high-pressure liquid chromatography columns, Anal. Chem. 80 (2008) 5009-5020.

[10] K. Kaczmarski, J. Kostka, W. Zapała, G. Guiochon, Modeling of thermal processes in high pressure liquid chromatography. I. Low pressure onset of thermal heterogeneity, J. Chromatogr. A 1216 (2009) 6560-6574. 
[11] K. Kaczmarski, F. Gritti, J. Kostka, G. Guiochon, Modeling of thermal processes in high pressure liquid chromatography. II. Thermal heterogeneity at very high pressures, J. Chromatogr. A 1216 (2009) 6575-6586.

[12] H. Poppe, J. Kraak, Influence of thermal conditions on the efficiency of high-performance liquid chromatographic columns, J. Chromatogr. A 282 (1983) 399-412.

[13] O. Dapremont, G. Cox, M. Martin, P. Hilaireau, H. Colin, Effect of radial gradient of temperature on the performance of large-diameter high-performance liquid chromatography columns. I. Analytical conditions, J. Chromatogr. A 796 (1998) 81-99.

[14] F. Gritti, G. Guiochon, Effects of the thermal heterogeneity of the column on chromatographic results, J. Chromatogr. A 1131 (2006) 151-165.

[15] G. Desmet, Theoretical calculation of the retention enthalpy effect on the viscous heat dissipation band broadening in high performance liquid chromatography columns with a fixed wall temperature, J. Chromatogr. A 1116 (2006) 89-96.

[16] F. Gritti, M. Martin, G. Guiochon, Influence of viscous friction heating on the efficiency of columns operated under very high pressures, Anal. Chem. 81 (2009) 3365-3384.

[17] M. Fallas, M. Hadley, D. McCalley, Practical assessment of frictional heating effects and thermostat design on the performance of conventional ( $3 \mu \mathrm{m}$ and $5 \mu \mathrm{m})$ columns in reversed-phase high-performance liquid chromatography, J. Chromatogr. A 1216 (2009) 3961-3969.

[18] F. Gritti, G. Guiochon, Optimization of the thermal environment of columns packed with very fine particles, J. Chromatogr. A 1216 (2009) 1353-1362.

[19] F. Gritti, M. Gilar, J. Jarrell, Quasi-adiabatic vacuum-based column housing for very highpressure liquid chromatography, J. Chromatogr. A 1456 (2016) 226-234.

[20] F. Gritti, M. Gilar, J. Jarrell, Achieving quasi-adiabatic thermal environment to maximize resolution power in very high-pressure liquid chromatography: Theory, models, and experiments, J. Chromatogr. A 1444 (2016) 86-98.

[21] J. C. Giddings, Dynamics of Chromatography, M. Dekker, New York, 1965. 
[22] U. Neue, M. Kele, Performance of idealized column structures under high pressure, J. Chromatogr. A 1149 (2007) 236-244.

[23] K. Horváth, J. Fairchild, K. Kaczmarski, G. Guiochon, Martin-synge algorithm for the solution of equilibrium-dispersive model of liquid chromatography, J. Chromatogr. A 1217 (2010) 8127-8135.

[24] P. Vajda, A. Felinger, Multilayer adsorption on fractal surfaces, J. Chromatogr. A 1324 (2014) 121-127.

[25] J. Xu, X. Jiang, J. Guo, Y. Chen, W. Yu, Competitive adsorption equilibrium model with continuous temperature dependent parameters for naringenin enantiomers on chiralpak ad column, J. Chromatogr. A 1422 (2015) 163-169.

[26] K. Horváth, A. Felinger, Influence of particle size and shell thickness of core-shell packing materials on optimum experimental conditions in preparative chromatography, J. Chromatogr. A 1407 (2015) 100-105.

[27] L. Jeong, R. Sajulga, S. Forte, D. Stoll, S. Rutan, Simulation of elution profiles in liquid chromatography i: Gradient elution conditions, and with mismatched injection and mobile phase solvents, J. Chromatogr. A 1457 (2016) 41-49. 


\section{Figure captions}

Figure 1: Retention factor of a solute at different positions in the column in case of $\Delta H=-10 \mathrm{~kJ} / \mathrm{mol}$ at different temperature gradients, $\Delta T \mathrm{~s}$ : blue $-10 \mathrm{~K}$, green $-20 \mathrm{~K}$, red $-30 \mathrm{~K}$, light blue $-40 \mathrm{~K}$, purple $-50 \mathrm{~K}$.

Figure 2: Relative migration velocity of a solute at different positions in the column in case of $\Delta H=-10 \mathrm{~kJ} / \mathrm{mol}$ at different temperature gradients, $\Delta T \mathrm{~s}$ : blue $-10 \mathrm{~K}$, green $-20 \mathrm{~K}$, red $-30 \mathrm{~K}$, light blue $-40 \mathrm{~K}$, purple $-50 \mathrm{~K}$.

Figure 3: Retention times as a function of change of molar enthalpy, $\Delta H$, and axial temperature.

Figure 4: Physical width of zones at the end of the column at different temperature and enthalpy changes.

Figure 5: Release velocities of solutes from the column at different temperature and molar enthalpy changes.

Figure 6: Chromatographic peak widths at different temperature and enthalpy changes.

Figure 7: Change of chromatographic efficiencies due to axial temperature gradient as a function of changes of molar enthalpy and total temperature in the column.

Figure 8: Change of resolution in effect of axial temperature gradient. 


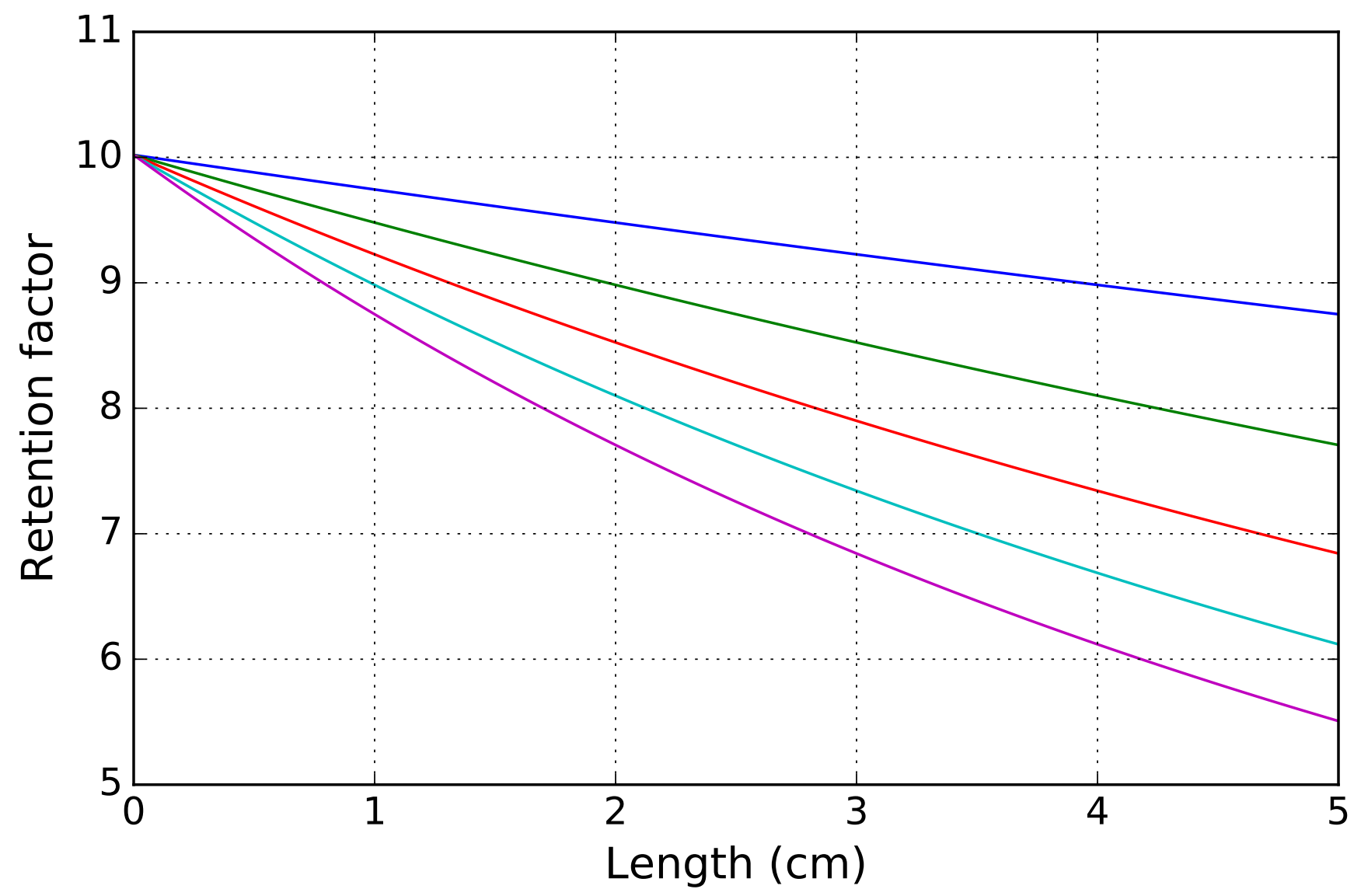




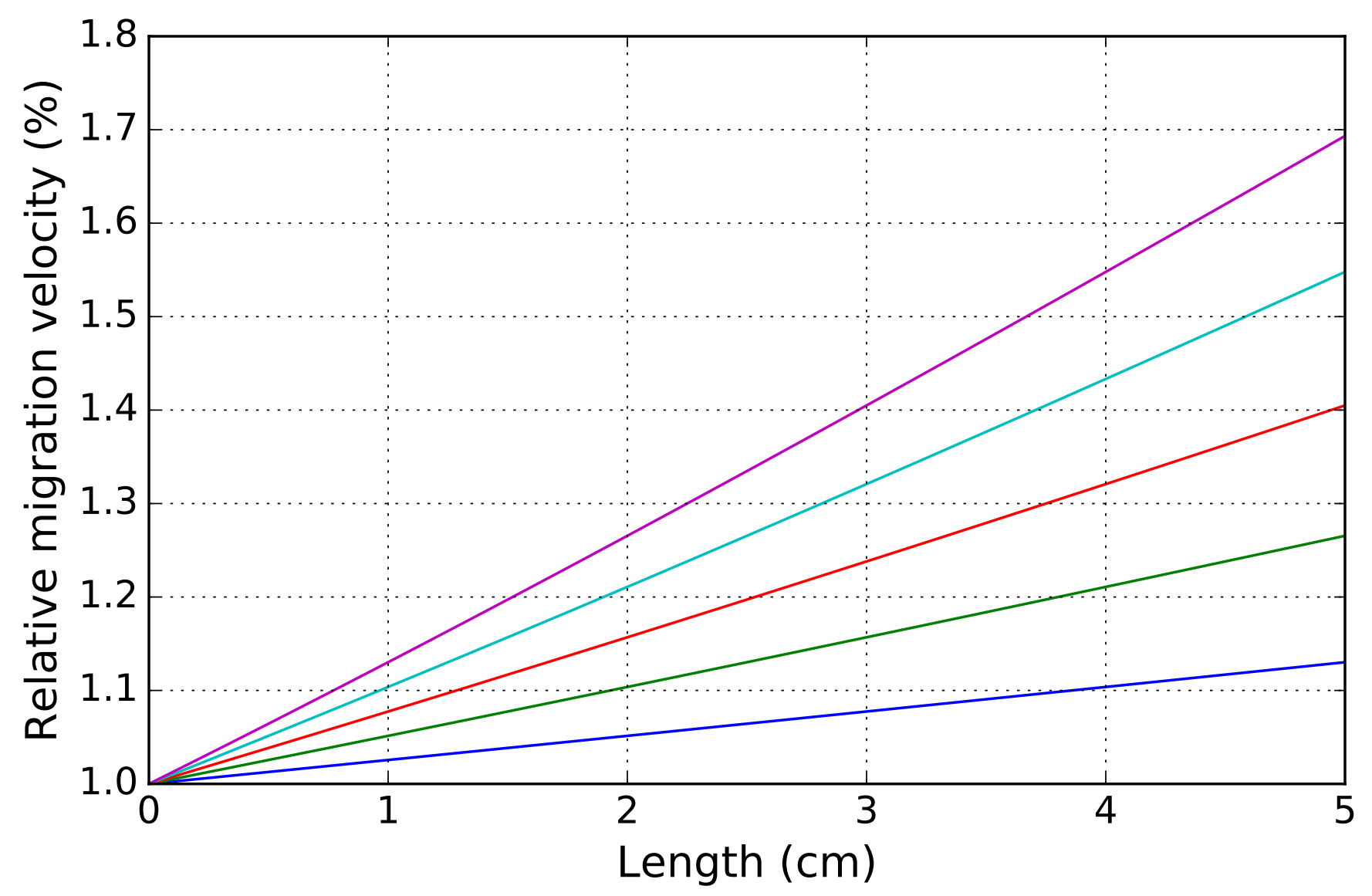




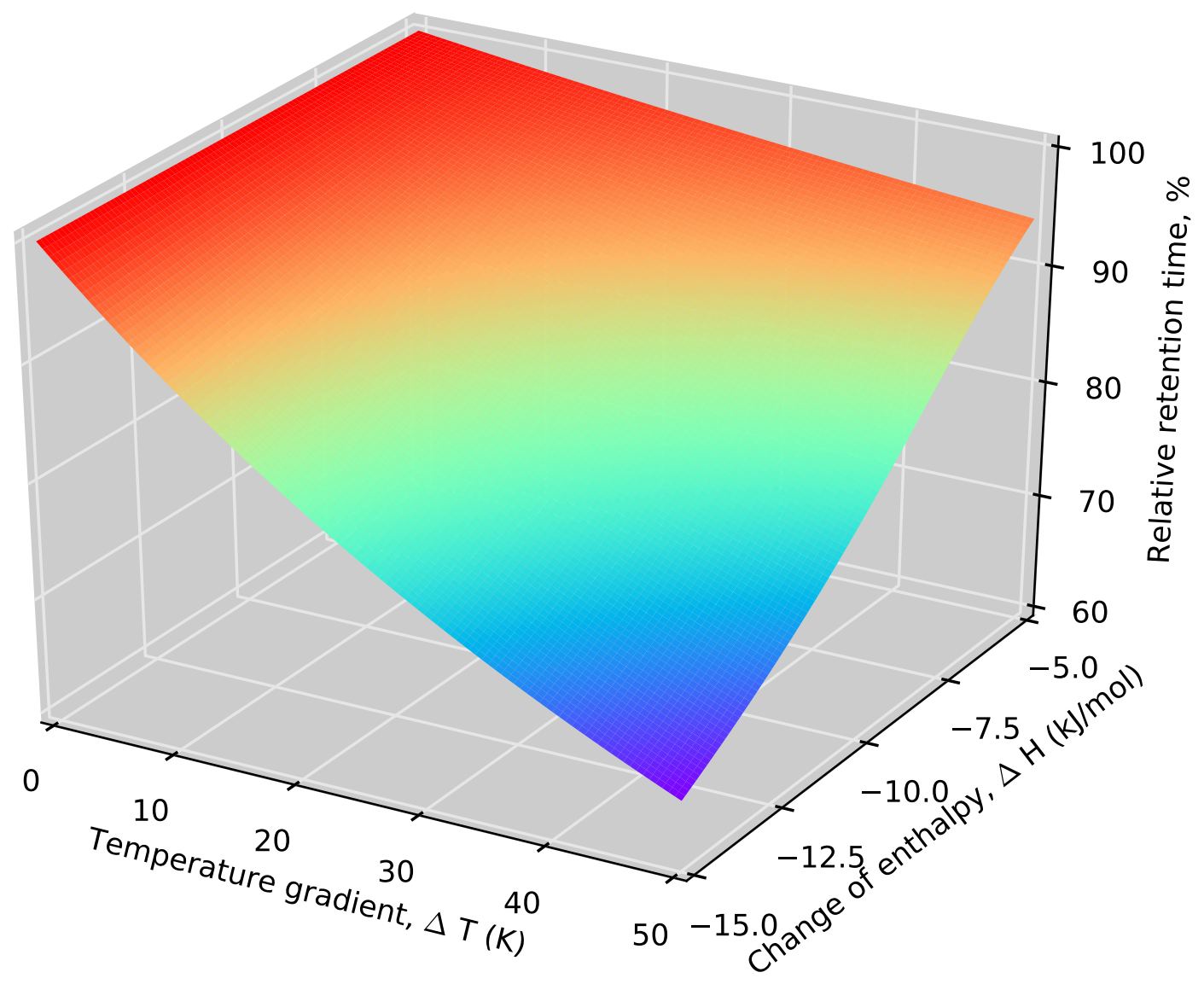




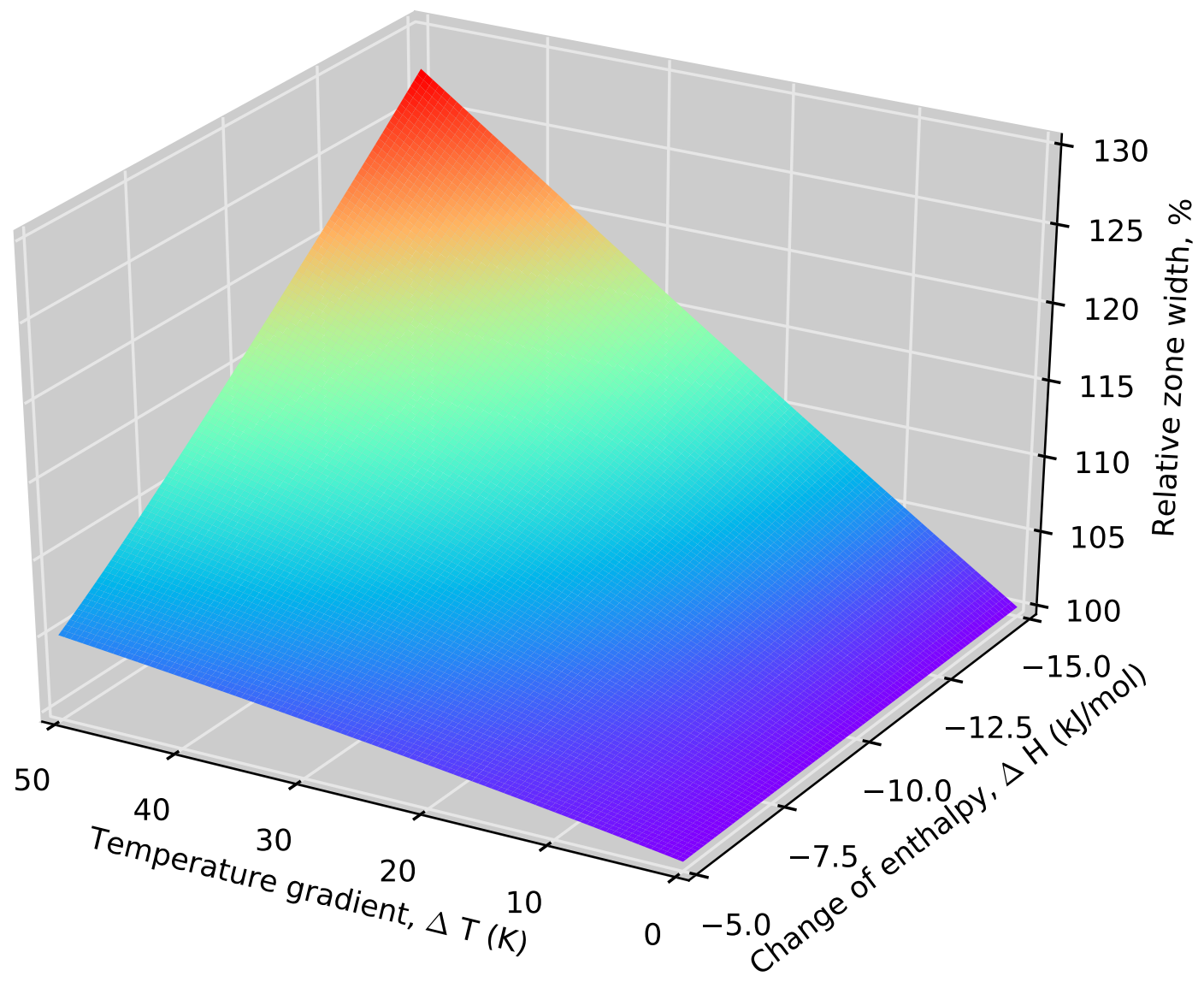




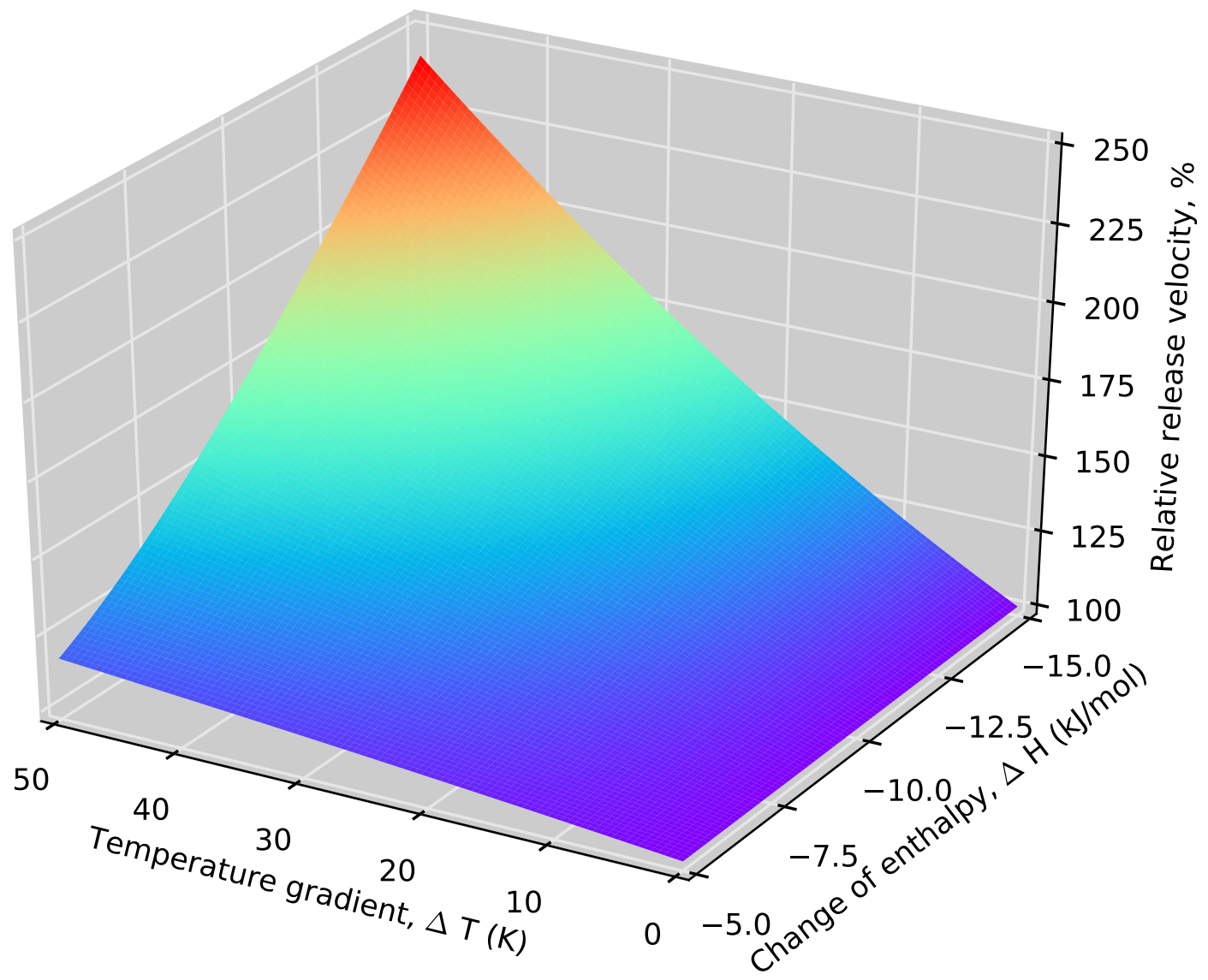




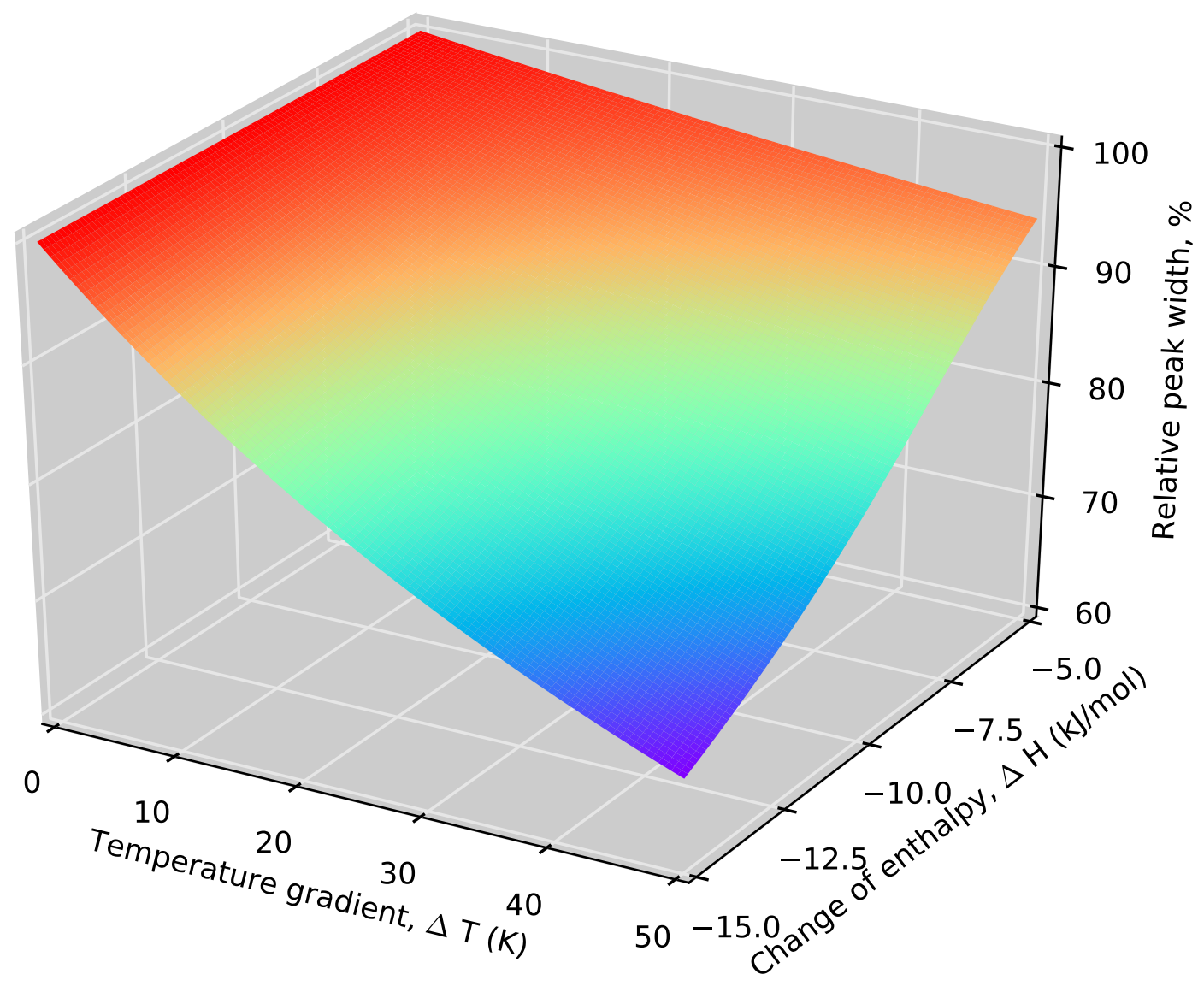




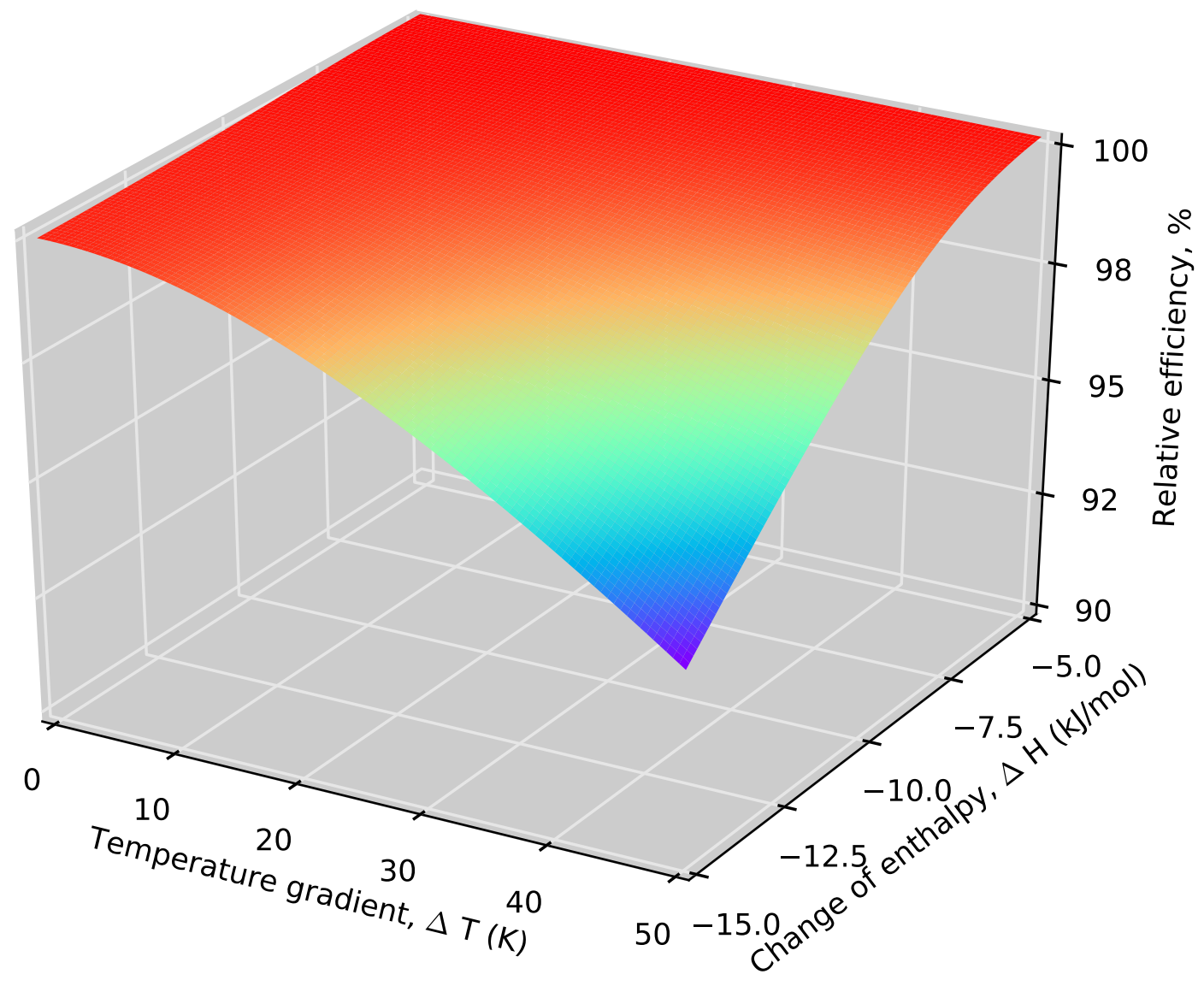




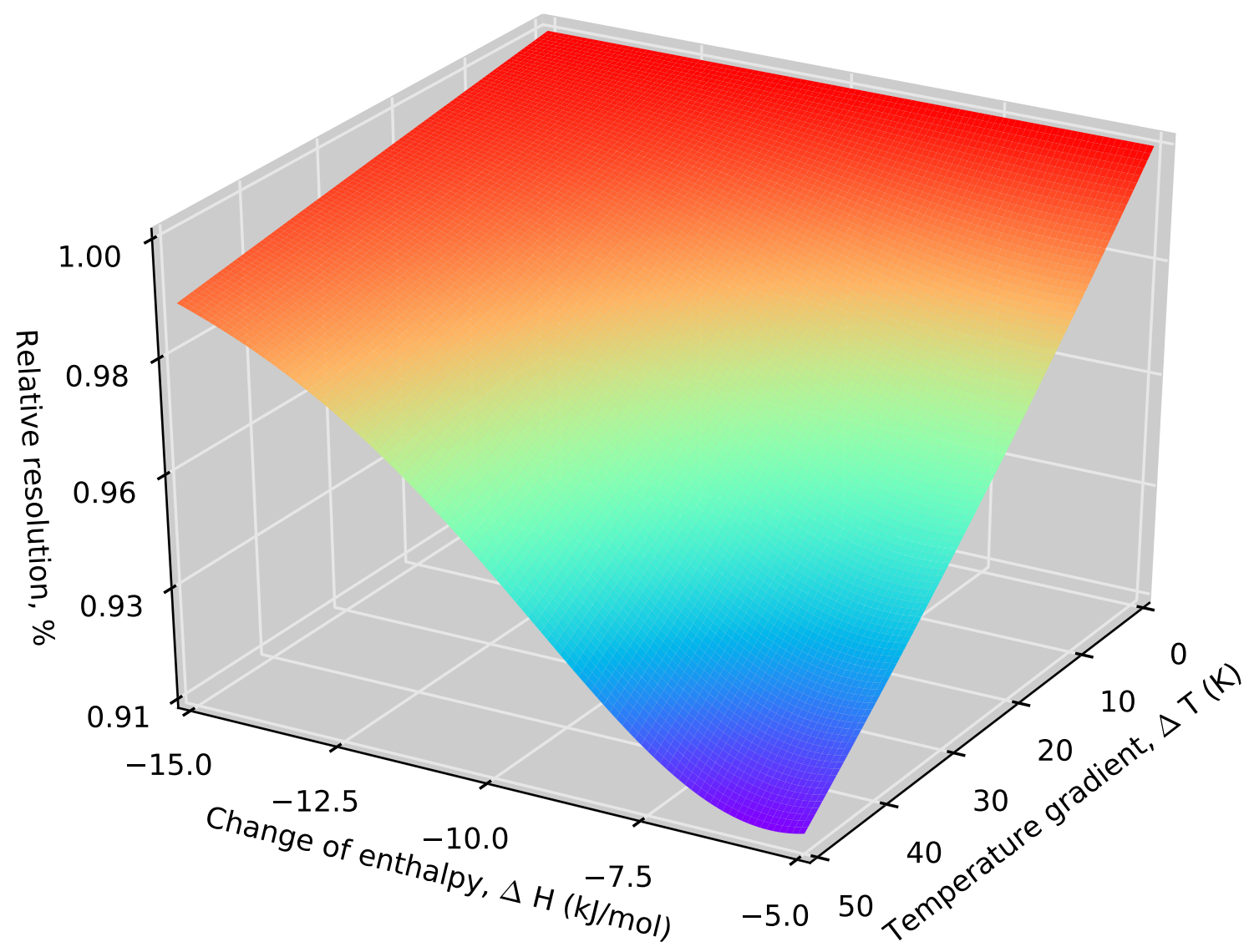

